\title{
Actividad larvicida de aceites esenciales y extractos de plantas colombianas frente a Culex quinquefasciatus (Díptera: Culicidae)
}

\section{Larvicidal activity of essential oils and extracts of colombian plants against Culex quinquefasciatus (Diptera: Culicidae)}

Doris Natalia Rosado-Solano

Universidad Santo Tomás

Bucaramanga, Colombia

natalia_enjoy@hotmail.com

Víctor Mario Jaramillo-Pérez Universidad Santo Tomás Bucaramanga

Bucaramanga, Colombia

qcavictor@gmail.com

Vladimir Valentinovich Kouznetsov

Universidad Industrial de Santander

Bucaramanga, Colombia

vkuznechnik@gmail.com

\author{
Ricardo Restrepo-Manrique \\ Universidad Santo Tomás \\ Bucaramanga, Colombia \\ restrepo.manrique@gmail.com
}

\author{
Carlos Eduardo Puerto-Galvis \\ Universidad Industrial de Santander \\ Bucaramanga, Colombia \\ cp_piru@hotmail.com
}

Leonor Yamile Vargas-Méndez

Universidad Santo Tomás Bucaramanga

Bucaramanga, Colombia

leyavar@gmail.com

\begin{abstract}
Resumen- los aceites esenciales y extractos de plantas, cultivadas de forma orgánica en la finca El Limonal Piedecuesta-Santander, Colombia, obtenidos por hidrodestilación asistida por microondas y percolación, se caracterizaron por cromatografía de gases acoplada a espectrometría de masas. La actividad insecticida se evaluó frente a larvas en tercer instar de Culex quinquefasciatus y en la enzima acetilcolinesterasa. La toxicidad se determinó en el modelo del pez cebra (Danio rerio). De ellos, el aceite esencial de Ruta graveolens fue el que presentó la mejor actividad larvicida, con $\mathrm{CL}_{50}$ $7.20 \pm 0.19 \mu \mathrm{g} / \mathrm{mL}$, una moderada inhibición de la enzima con IC $\mathrm{F}_{50} 44.98 \pm 1.1 \mu \mathrm{g} / \mathrm{mL}$ y moderada toxicidad con $\mathrm{CL}_{50} 13.33 \pm 0.59 \mathrm{mg} / \mathrm{L}$.
\end{abstract}

Palabras clave- Aceites esenciales, actividad larvicida, bioensayos en embriones cebra, Culex quinquefasciatus, extractos, inhibidores de acetilcolinesterasa.

Abstract- Essential oils and extracts obtained by microwave hydrodistillation and percolation from plants organically cultivated in the farm El Limonal Piedecuesta-Santander, Colombia, were characterized by Gas Chromatography coupled with Mass Spectrometry. The insecticidal activity against third instar larva of Culex quinquefasciatus and the inhibition activity of acetylcholinesterase of those compounds were studied. Also, the toxicity was evaluated against the zebrafish (Danio rerio). Our results show that the Ruta graveolens essential oil had the highest larvicidal activity with a LC $_{50}$ $7.20 \pm 0.19 \mu \mathrm{g} / \mathrm{mL}$, a moderate inhibition over the enzyme with an $I_{50} 44.98 \pm 1.1 \mu \mathrm{g} / \mathrm{mL}$ and a moderate toxicity with a $L C_{50} 13.33 \pm 0.59 \mathrm{mg} / \mathrm{L}$.

Keywords- Acetylcholinesterase inhibitors, Culex, quinquefasciatus, essential oils, extracts, larvicidal activity, zebrafish embryos bioassays.

\section{INTRODUCCIÓN}

Los pesticidas pueden ser considerados los milagros de la modernidad. Estos clústeres de átomos altamente estructurados y relativamente pequeños son unos de los principales agentes que han permitido disminuir la morbilidad y mortalidad por las infecciones transmitidas por diferentes plagas.

Muchas de las "moléculas milagro" pueden salvar vidas humanas, de manera indirecta, al ser usadas para el control de los vectores de enfermedades de importancia médica y veterinaria, al tiempo que se obtienen ganancias, por su venta, de billones de dólares por año; el desarrollo de 
cada uno de ellos es arduo y costoso, con enormes inversiones en su desarrollo, pero también con grandes beneficios.

Los mosquitos son conocidos como vectores de algunas enfermedades que son causadas por agentes patógenos. El Aedes. aegypti es distinguido por transmitir Dengue, Zika y Chikungunya [1]; la trasmisión de la malaria se da por el Anopheles. stephensi y la filariasis por Culex spp [2]. Actualmente existen más de 120 millones de personas que padecen filariasis, y más de 1400 millones en 73 países se encuentran en riesgo de padecer esta enfermedad.

Una forma de prevenir la infestación por mosquitos adultos es a través del uso de larvicidas organofosforados (temefos) [3] o de inhibidores del crecimiento (piriproxifen) [4]. El uso continuo de los insecticidas sintéticos ha ocasionado el desarrollo de resistencia en los mosquitos [5]. Se plantea que los aceites esenciales son excelentes fuentes de compuestos bioactivos que pueden actuar como larvicidas, reguladores del crecimiento, inhibidores de la oviposición y repelentes, además de ser compuestos de fácil degradación y más seguros para los organismos no-objetivos como el ser humano [6].

Por el interés de encontrar nuevas alternativas en el estudio de nuevas para el control de los mosquitos en su estado larval, en esta investigación se evaluaron aceites esenciales y extractos de plantas aromáticas y medicinales como posibles agentes larvicidas en Culex quinquefasciatus.

\section{MATERIALES Y MÉTODOS}

\subsection{Reactivos}

Los reactivos utilizados fueron: hexano, etanol, dimetilsulfóxido (DMSO), agua, (J.T Baker, grado analítico), hidrógeno fosfato de sodio, hidrógeno fosfato de potasio, Tween 20, yoduro de acetiltiocolina, ácido ditiobisnitrobenzoico, capsaicina, dihidrocapsaicina, acetilcolinesterasa de Electrophorus electricus, cloruro de sodio, cloruro de potasio, cloruro de calcio, sulfato de magnesio, temefos, y malation (Sigma-Aldrich).

\subsection{Material vegetal}

El material vegetal se cultivó en la finca El Limonal en el municipio de Piedecuesta (Santan- der, Colombia), con coordenadas geográficas: latitud $7^{\circ} 0.477^{\prime} \mathrm{N}$, longitud $73^{\circ} 3.205^{\prime} \mathrm{O}$ y altitud de $1020 \mathrm{msnm}$. La identificación taxonómica fue realizada en el Herbario Nacional del Instituto de Ciencias Naturales de la Universidad Nacional de Colombia:

Mentha piperita $L$. de la familia Lamiaceae No. COL 574691;

Ocimum americanum $L$. de la familia Lamiaceae No. COL 574690;

Cymbopogon citratus (DC.) Stapf de la familia Poaceae No. COL 574692;

Chenopodium ambrosioides $L$. de la familia Chenopodiaceae No. COL 574695;

Eucalyptus globulus Labill. de la familia Miyrtaceae No. COL 574696;

Ruta graveolens $L$. de la familia Rutaceae No. COL 574697;

Rosmarinus officinalis $L$. de la familia Lamiaceae No. COL 573968;

Capsicum chinense Jacq. de la familia Solanaceae No. COL 558072;

Dieffenbachia seguine (Jacq.) Schott. de la familia Araceae No. COL 557711;

Urera baccifera (L.). Gaudich. Ex Wedd de la familia Urticaceae No. COL 557323.

Para la obtención de los AE se emplearon hojas, tallos y flores; para el extracto de $C$. chinense se empleó el fruto fresco, para el de $D$. seguine las hojas secas y para el de $U$. baccifera tallos y hojas secas. Tanto para los AE como los extractos se emplearon plantas de aspecto sano y robusto, en estado de madurez y floración.

\subsection{Obtención de los extractos}

Los extractos se obtuvieron por percolación, usando un equipo de maceración dinámica, en la cual el material vegetal se colocó en contacto con $150 \mathrm{~mL}$ de etanol durante tres días con agitación continúa durante 4 horas cada 24 horas. A los extractos alcohólicos se les separó el disolvente en un rotaevaporador, y las trazas se eliminaron conectando los balones a un manifold unido a una bomba de alto vacío $(5 \mathrm{mmHg}$ ) durante cuatro horas. Todas las extracciones se hicieron por triplicado. 


\section{4 Obtención de los aceites esenciales}

Los aceites esenciales se obtuvieron mediante hidrodestilación asistida por microondas. En un balón fondo redondo, equipado con una trampa Dean-Stark y dos condensadores tipo rosario y en espiral, se adicionó el material vegetal fresco (hojas, tallos y flores) de cada una de las plantas, en trozos pequeños, se les adicionó $500 \mathrm{~mL}$ de agua, la mezcla se calentó a ebullición, durante $75 \mathrm{mi}-$ nutos en tres sesiones de 25 minutos cada una.

\subsection{Cromatografía de gases-espectrometría de masas}

Los extractos se analizaron en un cromatógrafo de gases Agilent Technologies 7890A acoplado a un detector selectivo de masas Agilent Technologies 5975C, con inyector split/splitless (relación split 1:10) y columna HP-1MS (30 m x $0.25 \mathrm{~mm}$ D.I. $\times 0.25 \mu \mathrm{m}$ df) con fase estacionaria $100 \%$-fenil-poli(dimetilsiloxano).

La rampa de calentamiento utilizada en el horno cromatográfico fue: $50^{\circ} \mathrm{C}(5 \mathrm{~min}) @ 5^{\circ} \mathrm{C} / \mathrm{min}$ hasta $200^{\circ} \mathrm{C}(5 \mathrm{~min}) \mathrm{y} @ 10^{\circ} \mathrm{C} / \mathrm{min}$ hasta $260^{\circ} \mathrm{C}$ (20 min). Los espectros de masas se obtuvieron por ionización electrónica $(70 \mathrm{eV})$ en un analizador cuadrupolar con rango de masas m/z 40-400, en el modo full scan. Las temperaturas de la cámara de ionización y de la línea de transferencia se mantuvieron en 230 y $285^{\circ} \mathrm{C}$, respectivamente. Los datos cromatográficos y espectroscópicos se procesaron con el software de Agilent Technologies MSD ChemStation G1701 (Versión EA E.02.02.1431).

La identificación de los componentes de los aceites esenciales y extractos fue realizada comparando los espectros de masas obtenidos, con los reportados en las bases de datos Adams [7] Wiley 138 [8], y NISTO2 [9].

\subsection{Ensayo de actividad insecticida}

Para implementar el bioterio se recolectaron los huevos de Culex quinquefasciatus en un colector de aguas lluvias en Bucaramanga, Santander, con coordenadas geográficas: latitud $7^{\circ} 6$ ' $11.3^{\prime \prime} \mathrm{N}$, longitud $73^{\circ} 6^{\prime} 52.1^{\prime \prime} \mathrm{W}$, y altura $947 \mathrm{msnm}$. El 10\% de los huevos y larvas eclosionadas fueron enviados al Instituto de Salud de Bucaramanga para su caracterización entomológica.
La actividad larvicida se realizó siguiendo la metodología de la Organización Mundial de la Salud [10]including bacterial larvicides and insect growth regulators (IGRs. Las concentraciones de los aceites esenciales y extractos se probaron de forma seriada desde 1000 hasta 1 ppm; un control. Para disolver el malatión se utilizó agua y para las muestras etanol.

Se colocaron lotes de 25 larvas en vasos plásticos desechables y se les adicionó delicadamente $33 \mathrm{~mL}$ de la solución correspondiente, se dispusieron controles positivos (agua), y controles negativos (etanol), a la concentración NOAEL, que es aquella a la que no se observa ningún efecto adverso sobre las larvas (2\%).

Pasadas 72 horas de exposición se leyó la mortalidad, determinando el daño, muerte o alteración fisiológica en las larvas expuestas. Fueron consideradas muertas aquellas larvas a las que no se les pudo inducir el movimiento. Las larvas moribundas fueron adicionadas al número de larvas muertas; se consideraron como moribundas aquellas larvas incapaces de elevarse a la superficie o que no mostraron el buceo característico cuando son perturbadas.

Cada ensayo se hizo por triplicado, en semanas diferentes, con un fotoperiodo de 12 horas de luz y 12 horas de oscuridad. A partir de los porcentajes de mortalidad obtenidos, los datos se procesaron en función de la concentración, calculando los parámetros estadísticos con el método de regresión logarítmica en el programa BioStat 2009, a partir del cual se obtuvieron las Concentraciones Letales Media y $\mathrm{CL}_{95}$.

\subsection{Ensayo de inhibición de la enzima acetil- colinesterasa}

Para determinar la inhibición de la acetilcolinesterasa por los extractos y aceites, se utilizó la metodología de Ellman [11]homogenates, cell suspensions, etc., has been described. The enzyme activity is measured by following the increase of yellow color produced from thiocholine when it reacts with dithiobisnitrobenzoate ion. It is based on coupling of these reactions: The latter reaction is rapid and the assay is sensitive (i.e. a 10 $\mu 1$ sample of blood is adequate. Se colocaron 50 $\mu \mathrm{L}$ de una solución del extracto o aceite esencial (en concentraciones seriadas desde 1000 
hasta $0.5 \mathrm{ppm})$, disuelto en buffer fosfato salino de $\mathrm{pH} 7.5$ y $50 \mu \mathrm{L}$ de la AChE $(0.25 \mathrm{U} / \mathrm{mL})$. La placa se incubó a temperatura ambiente por 30 minutos y se adicionaron $100 \mu \mathrm{L}$ de la solución sustrato de pH $7.5\left[\mathrm{Na}_{2} \mathrm{HPO}_{4} 0.04 \mathrm{M}\right.$, ácido 2,2 $2^{-}$-dinitro-5,5'-ditio benzoico $0.2 \mathrm{mM}$, yoduro de acetiltiocolina $0.24 \mathrm{mM}$. A los cinco minutos de iniciada la reacción se leyó la absorbancia, a $412 \mathrm{~nm}$, en un lector de microplacas VERSAmax. Se utilizó galantamina como compuesto de referencia. Los ensayos se realizaron por triplicado; los cálculos de las $\mathrm{IC}_{50}$ y las gráficas se hicieron con el software SoftMax Pro 5.2 de Molecular Devices.

\subsection{Ensayo de toxicidad y análisis de los cambios fenotípicos de los extractos etanó- licos empleando el modelo embrionario del pez cebra}

Se usaron los procedimientos reportados por Ali [12], Eggert [13], Peterson [14] Rubinstein [15] y adaptados por Puerto \& Kouznetsov [16].

Especímenes adultos del pez cebra (Danio rerio), de ambos sexos y de tipo salvaje, fueron separados en tanques de acuerdo con su género a $26 \pm 2{ }^{\circ} \mathrm{C}$ bajo fotoperíodos naturales de luz y oscuridad. Los peces fueron alimentados dos veces por día y la calidad del agua fue monitoreada semanalmente, con el fin de adaptar a los peces a estas condiciones por dos semanas, antes de iniciar los experimentos.

Para la reproducción de los peces adultos, pequeños tanques de apareamiento fueron acondicionados en la tarde previa al experimento, donde en cada uno de estos tanques se ubicaron tres machos y una hembra. Estos tanques fueron aislados, sin ninguna perturbación de ruido o luz, hasta la mañana siguiente, cuando el apareamiento natural ocurrió.

Los peces adultos fueron reintegrados a sus respectivos tanques y los embriones fueron recolectados, lavados dos veces con buffer de Holtfreter (compuesto por $5 \mathrm{mM} \mathrm{NaCl}, 0.17 \mathrm{mM} \mathrm{KCl}$, $0.4 \mathrm{mM} \mathrm{CaCl}_{2}$ y $0.16 \mathrm{mM} \mathrm{MgSO}_{4}$, al cual nombramos E3), y transferidos a una caja Petri. Los embriones fueron examinados periódicamente a través de un microscopio de disección con el fin de remover aquellos embriones muertos, no fer- tilizados, malformados o retrasados en su desarrollo. Durante este período, los embriones fueron mantenidos en una incubadora a $28 \pm 2{ }^{\circ} \mathrm{C}$ bajo fotoperíodos naturales de luz y oscuridad.

Los embriones seleccionados a 24 horas posteriores a la fertilización (hpf) fueron transferidos cuidadosamente desde la caja Petri a una microplaca de 96 pozos, ubicando un embrión y 200 $\mu \mathrm{L}$ de E3 por pozo.

\subsubsection{Determinación de la $\mathrm{CL}_{50}$ en embrio- nes del pez cebra}

Para este experimento se emplearon 72 embriones en total, para evaluar la toxicidad de cada extracto con el fin de realizar tres experimentos independientes en tres diferentes placas, en cada una de ellas cada extracto fue evaluado tres veces en cada placa.

En cada placa se evaluó una serie geométrica de concentraciones, iniciando en 300 y finalizando en $4.68 \mu \mathrm{g} / \mathrm{mL}$. La mortalidad acumulada fue monitoreada en un microscopio de disección 72 horas después de la exposición química (96 hpf). Se determinó la $\mathrm{CL}_{50}$ (expresada en $\mathrm{mg}$ de extracto/mL de solución) mediante el análisis de la Regresión Probit con el programa SPSS para Windows versión 19.0. Los datos se expresan con el error estándar de la media de los tres experimentos realizados en triplicado.

\subsubsection{Análisis de los cambios fenotípicos usando el modelo embrionario del pez cebra}

Los extractos de C. chinense, U. baccifera, $C$. officinalis fueron diluidos en el medio E3 con $2 \%$ $\mathrm{v} / \mathrm{v}$ de DMSO y se prepararon alícuotas de 200 $\mu \mathrm{L}$ en una placa de 96 pozos en concentraciones de $100,50,25,12.5,6.25,3.12,1.56,0.78$ y $0.39 \mu \mathrm{g} / \mathrm{mL}$.

Se utilizaron cuatro embriones como control, cada uno expuesto al medio E3 con un 2\% v/v de DMSO. La placa embrionaria fue incubada a $28 \pm 2^{\circ} \mathrm{C}$ bajo fotoperíodos naturales de luz y oscuridad, y los embriones fueron examinados a 48, 72 y 96 hpf utilizando un estéreomicroscopio trinocular OPTIKA (versión del modelo: SZM-1). Cada uno de estos experimentos fue realizado por duplicado. 


\section{RESULTADOS}

\subsection{Obtención de aceites esenciales y ex- tractos}

Los aceites esenciales de las plantas se aislaron con un rendimiento en peso fresco de: $1.6 \%$ para $R$. graveolens; $0.9 \%$ para 0 . americanum; $0.14 \%$ para M. piperita; $1.30 \%$ para E. globulus; $0.05 \%$ para $R$. officinalis; $0.15 \%$ para C. ambrosoides y $0.25 \%$ para C. citratus. Las características físicas de los aceites mostraron ser insolubles en agua, solubles en etanol y dimetilsulfoxido, de tonalidad amarillo pálido tenue para $R$. graveolens y M. piperita e incoloro para 0 . americanum.

Los extractos de las plantas se aislaron con un rendimiento de: $1.3 \%$ para $D$. seguine; $0.4 \%$ para $U$. baccifera y $0.6 \%$ para $C$. chinense.

\subsection{Cromatografía de gases-espectrometría de masas}

El análisis por CG-MS de la composición química, y la cantidad relativa de componentes de los extractos y aceites esenciales se muestran en las tablas I y II, respectivamente. Estos extractos presentaron compuestos químicos similares en su composición, por ejemplo, la amida docosenamida se presentó en el extracto $C$. chinense $D$. seguine y $U$. baccifera; el neofitadieno se presentó en los últimos dos extractos.

TABLA I

COMPOSICIÓN QUÍMICA DE LOS EXTRACTOS DE C. CHINENSE, D SEGUINE Y U. BACCIFERA

\begin{tabular}{|l|c|c|c|}
\hline \multirow{2}{*}{ Compuesto } & \multicolumn{3}{c|}{ Cantidad relativa, \% } \\
\cline { 2 - 4 } & $\begin{array}{c}\text { Capsicum } \\
\text { chinense }\end{array}$ & $\begin{array}{c}\text { Dieffen- } \\
\text { bachia } \\
\text { seguine }\end{array}$ & $\begin{array}{c}\text { Urera } \\
\text { baccifera }\end{array}$ \\
\hline Palmitato de etilo & 2.1 & - & - \\
\hline Linoleato de etilo & 3.2 & - & - \\
\hline Capsaicina & 74.1 & - & - \\
\hline Dihidrocapsaicina & 8.9 & - & - \\
\hline Docosenamida & 11.7 & 11.3 & 10.0 \\
\hline $\begin{array}{l}\text { Neofitadieno } \\
\text { (isómeros) }\end{array}$ & - & 41.0 & 55.3 \\
\hline Fitol & - & 20.3 & 11.9 \\
\hline Fiteno & - & 8.1 & 9.1 \\
\hline Total & 100 & 80.7 & 86.3 \\
\hline
\end{tabular}

Fuente: Los autores.
Los $A E$ presentaron una composición química muy variada (ver tabla II), el eucaliptol, el $\alpha$-pineno, y el $\beta$-cariofileno fueron los metabolitos secundarios, tipo terpenoides que se presentaron en dos o más de los AE obtenidos.

\subsection{Estudio para determinar la actividad insecticida de los aceites esenciales y ex- tractos}

Los AE de R. graveolens $\left(\mathrm{CL}_{50} 7.20 \pm 0.19 \mu \mathrm{g} /\right.$ $\mathrm{mL})$, O. americanum $\left(\mathrm{CL}_{50} 16.8 \pm 0.11 \mu \mathrm{g} / \mathrm{mL}\right)$ y C. ambrosioides $\left(\mathrm{CL}_{50} 25.4 \pm 0.5 \mu \mathrm{g} / \mathrm{mL}\right)$ son altamente activos, con concentraciones letales medias menores a $50 \mu \mathrm{g} / \mathrm{mL}$; los $\mathrm{AE}$ de $M$. piperita $\left(\mathrm{CL}_{50} 53.9 \pm 0.9 \mu \mathrm{g} / \mathrm{mL}\right)$, E. globulus $\left(\mathrm{CL}_{50} 57.7 \pm 1.2\right.$ $\mu \mathrm{g} / \mathrm{mL})$ y C. citratus $\left(\mathrm{CL}_{50} 75.5 \pm 3.1 \mu \mathrm{g} / \mathrm{mL}\right)$ son activos; con concentraciones letales medias entre $50-100 \mu \mathrm{g} / \mathrm{mL}$ y el AE de R. officinalis tiene moderada actividad con $\mathrm{CL}_{50} 121.8 \pm 0.9 \mu \mathrm{g} / \mathrm{mL}$. Por otro lado, los extractos de $D$. seguine $\left(\mathrm{CL}_{50}\right.$ $693.5 \pm 33 \mu \mathrm{g} / \mathrm{mL})$, U. baccifera $\left(\mathrm{CL}_{50} 873.9 \pm 39\right.$ $\mu \mathrm{g} / \mathrm{mL})$ y C. chinense $\left(\mathrm{CL}_{50} 1056 \pm 4.4 \mu \mathrm{g} / \mathrm{mL}\right)$ son considerados como poco activos frente a las larvas de Culex quinquefasciatus en tercer instar. Las $\mathrm{CL}_{50}$ y $\mathrm{CL}_{95}$ de cada uno de ellos se muestran en la tabla III.

Las bioactividades dependen en gran medida de la composición química y puede verse afectada por la zona geográfica en donde se cultive la planta, y las condiciones climáticas. Algunos ejemplos de esto son los AE de Ocimum americanum, Chenopodium ambrosioides y Mentha piperita.

En Brasil, el AE de O. americanum quimiotipo cinamato de metilo (70.9\%) mostró una $\mathrm{CL}_{50}$ de $67 \mu \mathrm{g} / \mathrm{mL}$, transcurridas las 24 horas de exposición, frente a larvas de Aedes aegypti [17]. Con lo cual el AE es considerado como activo frente a esta cepa del laboratorio de la Secretaría de Salud del estado Ceará, Brasil. En esta investigación el $A E$ aislado es quimotipo linalol (43.3\%), y es altamente activo $\left(\mathrm{CL}_{50} 16.8 \mu \mathrm{g} / \mathrm{mL}\right)$ para la cepa estudiada de Culex quinquefasciatus.

EI AE de C. ambrosioides es quimiotipo limoneno (23.8\%), y presentó una $\mathrm{CL}_{50}$ de $25.4 \mathrm{\mu g} /$ $\mathrm{mL}$. La literatura reportó el quimiotipo $\alpha$-terpineol (73.9\%) como insecticida en larvas de Aedes aegypti, con $\mathrm{CL}_{50}$ de $35 \mu \mathrm{g} / \mathrm{mL}$ [18]. Siendo ambos quimiotipos altamente activos sobre las especies de mosquitos evaluados. 
TABLA II

COMPOSICIÓN QUÍMICA DE LOS ACEITES ESENCIALES OBTENIDOS POR MW-HD

\begin{tabular}{|c|c|c|c|c|c|c|c|}
\hline \multirow[b]{2}{*}{ Compuesto } & \multicolumn{7}{|c|}{ Composición relativa (\%) } \\
\hline & $\begin{array}{l}\text { Ocimum ame- } \\
\text { ricanum }\end{array}$ & $\begin{array}{l}\text { Eucalyptus } \\
\text { globulus }\end{array}$ & $\begin{array}{c}\text { Rosmarinus } \\
\text { officinalis }\end{array}$ & $\begin{array}{l}\text { Chenopodium } \\
\text { ambrosoides }\end{array}$ & $\begin{array}{l}\text { Metha } \\
\text { piperita }\end{array}$ & $\begin{array}{l}\text { Ruta graveo- } \\
\text { lens }\end{array}$ & $\begin{array}{l}\text { Cymbopogon } \\
\text { citratus }\end{array}$ \\
\hline Linalol & 43.3 & - & - & - & - & - & - \\
\hline $\begin{array}{l}\text { Trans-cinamato de } \\
\text { metilo }\end{array}$ & 14.4 & - & - & - & - & - & - \\
\hline Estragol & 11.5 & - & - & - & - & - & - \\
\hline Tau-Cadino & 9.30 & - & - & - & - & - & - \\
\hline Eucaliptol & 4.80 & 54.9 & 7.85 & - & 6.00 & - & - \\
\hline Eugenol & 2.19 & - & - & - & - & - & - \\
\hline$\alpha$-Pineno & - & 18.6 & 7.66 & - & - & - & - \\
\hline$\alpha$-Terpineol & - & 3.91 & - & - & - & - & - \\
\hline$\alpha$-Acetato de terpinil & - & 3.31 & - & - & - & - & - \\
\hline Aromadendreno & - & 2.57 & - & - & - & - & - \\
\hline Globulol & - & 2.32 & - & - & - & - & - \\
\hline p-Cimeno & - & - & - & 11.8 & - & - & - \\
\hline$\beta$-Cariofileno & - & - & 49.7 & - & 2.84 & - & - \\
\hline Alcanfor & - & - & 6.80 & - & - & - & - \\
\hline Camfeno & - & - & 3.55 & - & - & - & - \\
\hline Borneol & - & - & 2.71 & - & - & - & - \\
\hline Limoneno & - & - & - & 23.8 & - & - & - \\
\hline p-Cimeno & - & - & - & 11.8 & - & - & - \\
\hline$\alpha$-Terpineno & - & - & - & 11.5 & - & - & - \\
\hline $\begin{array}{l}\text { Trans-p-menta- } \\
\text { 2.8-dienol }\end{array}$ & - & - & - & 8.43 & - & - & - \\
\hline Carvacrol & - & - & - & 5.16 & - & - & - \\
\hline $\begin{array}{l}\text { Trans-p-Menta- } \\
\text { 1(7).8-dien-2-ol }\end{array}$ & - & - & - & 4.83 & - & - & - \\
\hline Mentol & - & - & - & - & 27.0 & - & - \\
\hline Mentona & - & - & - & - & 15.7 & - & - \\
\hline Acetato de mentilo & - & - & - & - & 11.33 & - & - \\
\hline Mentofurano & - & - & - & - & 6.34 & - & - \\
\hline 2-Nonanona & - & - & - & - & - & 35.4 & - \\
\hline 2-Undecanona & - & - & - & - & - & 30.5 & - \\
\hline Triterpeno & - & - & - & - & - & 6.11 & - \\
\hline $\begin{array}{l}\text { Derivado ácido } \\
\text { benzodioxon }\end{array}$ & - & - & - & - & - & 3.92 & - \\
\hline 2-Decanona & - & - & - & - & - & 3.44 & - \\
\hline Geijereno & - & - & - & - & - & 2.58 & - \\
\hline Geranial & - & - & - & - & - & - & 30.3 \\
\hline Neral & - & - & - & - & - & - & 23.9 \\
\hline$\beta$-Mirceno & - & - & - & - & - & - & 6.79 \\
\hline Ácido Genérico & - & - & - & - & - & - & 6.39 \\
\hline $\begin{array}{l}\text { Monoterpeno } \\
\text { oxigenado }\end{array}$ & - & - & - & - & - & - & 4.00 \\
\hline $\begin{array}{l}\text { 3,7-Dimetil-2,6 } \\
\text {-octadien-1-ol }\end{array}$ & - & - & - & - & - & - & 3.86 \\
\hline Total & 85.5 & 85.6 & 78.3 & 65.5 & 69.2 & 81.9 & 75.2 \\
\hline
\end{tabular}

Fuente: Los autores. 
TABLA III

ACTIVIDAD LARVICIDA E INHIBICIÓN DE LA ACHE DE LOS ACEITES ESENCIALES Y EXTRACTOS

\begin{tabular}{|c|c|c|c|c|c|c|c|}
\hline & & \multicolumn{4}{|c|}{ Actividad larvicida } & \multicolumn{2}{|c|}{ Inhibición AChE } \\
\hline Descripción & Planta & $\begin{array}{l}\mathrm{CL}_{50} \pm \mathrm{SD} \\
(\mu \mathrm{g} / \mathrm{mL})\end{array}$ & $\mathrm{CV} \%$ & $\begin{array}{l}\mathrm{CL}_{95} \pm \mathrm{SD} \\
(\mu \mathrm{g} / \mathrm{mL})\end{array}$ & $\mathrm{CV} \%$ & $\begin{array}{l}I_{50} \pm S D \\
(\mu g / m L)\end{array}$ & CV\% \\
\hline Aceite esencial & Ruta graveolens & $7.20 \pm 0.19$ & 2.7 & $19.6 \pm 0.16$ & 0.8 & $44.98 \pm 11$ & 2.4 \\
\hline Aceite esencial & Ocimum americanum & $16.8 \pm 0.11$ & 0.7 & $250.9 \pm 0.2$ & 0.1 & $35.04 \pm 0.7$ & 1.9 \\
\hline Aceite esencial & $\begin{array}{c}\text { Chenopodium ambro- } \\
\text { sioides }\end{array}$ & $25.4 \pm 0.5$ & 1.9 & $49.6 \pm 0.4$ & 0.8 & $45.12 \pm 1.1$ & 2.4 \\
\hline Aceite esencial & Mentha piperita & $53.9 \pm 0.9$ & 1.6 & $103.5 \pm 0.9$ & 0.8 & $47.39 \pm 0.2$ & 0.4 \\
\hline Aceite esencial & Eucalyptus globulus & $57.7 \pm 1.2$ & 2.1 & $140.4 \pm 0.5$ & 0.3 & $31.25 \pm 0.7$ & 2.2 \\
\hline Aceite esencial & Cymbopogon citratus & $75.5 \pm 3.1$ & 4.1 & $147.2 \pm 1.6$ & 1.1 & $46.07 \pm 1.9$ & 4.1 \\
\hline Aceite esencial & Rosmarinus officinalis & $121.8 \pm 0.9$ & 0.8 & $246.7 \pm 0.4$ & 0.2 & $63.47 \pm 2.5$ & 2.4 \\
\hline Extracto & Dieffenbachia seguine & $693.5 \pm 33$ & 4.7 & $1303 \pm 59$ & 4.5 & $19.31 \pm 0.4$ & 2.4 \\
\hline Extracto & Urera baccifera & $873.9 \pm 39$ & 4.5 & $1729 \pm 39$ & 2.3 & $37.02 \pm 0.9$ & 2.7 \\
\hline Extracto & Capsicum chinense & $1056 \pm 4.4$ & 0.4 & $2126 \pm 11$ & 0.5 & $18.80 \pm 0,4$ & 2.1 \\
\hline- & Malatión & $0.98 \pm 0.06$ & 5.8 & $8.54 \pm 0.20$ & 2.4 & - & - \\
\hline- & Galantamina & - & - & - & - & $0.30 \pm 0.01$ & 3.5 \\
\hline
\end{tabular}

Fuente: Los autores.

Se han encontrado reportes de actividad larvicida de algunas plantas del género Ruta. Para el caso del AE de Ruta chalepensis se observó variación en la composición química, según su forma de cultivo. Para las plantas silvestres el metabolito mayoritario fue la 2-nonanona (39.3\%); para las plantas cultivadas en vivero, el metabolito mayoritario fue 2-undecanona (37.4\%) [19]. Este cambio en el quimiotipo generó una leve variación en la actividad larvicida, siendo más activo el quimiotipo 2-undecanona con una $\mathrm{CL}_{50}$ de $33.18 \mu \mathrm{g} /$ $\mathrm{mL}$ que el quimiotipo 2-nonanona $\mathrm{CL}_{50} 35.66 \mu \mathrm{g} /$ $\mathrm{mL}$ sobre larvas de Aedes albopictus. Para la Ruta graveolens cultivada de forma orgánica se encontró que su componente mayoritario es la 2-nonanona (35.4\%) y su concentración letal media frente a las larvas de Culex quinquefasciatus fue de $7.20 \mu \mathrm{g} / \mathrm{mL}$, siendo el AE con mejor actividad.

Seis AE de Mentha piperita (cultivadas en Gran Bretaña, Rusia y República Checa) han sido estudiados sobre larvas en tercer instar de Culex quinquefasciatus [20]. Estos AE no presentaron el metabolito secundario acetato de mentilo; el cual se mostró en un $11.33 \%$ en las plantas cultivadas en la finca El Limonal. De estos seis AE, tres presentaron una composición similar a la mostrada por el AE de Mentha piperita que se expone en la tabla II. Estos tres $\mathrm{AE}$ presentaron $\mathrm{CL}_{50}$ variables, oscilando entre 83 y $104 \mu \mathrm{g} / \mathrm{mL}$ transcurridas las 24 horas de exposición. Para el caso de las larvas de Culex colectadas en Bucaramanga, la $\mathrm{CL}_{50}$ fue de $53.9 \mu \mathrm{g} / \mathrm{mL}$ tras 72 horas del ensayo.

Igualmente, se reportó el efecto que presentan los AE de varias especies de Eucalyptus (globulus ssp. Maidenii, globulus ssp. globulus) cultivados en Argentina, sobre larvas de Aedes aegypti (cepa CIPEIN) [22]. En ambos aceites, el 1,8-cienol o eucaliptol fue el metabolito mayoritario con porcentajes de 77.91 y $76.66 \%$, respectivamente. Para el caso del Eucalyptus globulus de esta investigación, el eucaliptol se presentó en un 54.9\%, siendo este el compuesto mayoritario.

Los porcentajes de mortalidad de las variedades de eucaliptos argentinos no superaron el $20 \%$, siendo más activo la variedad Eucalyptus globulus ssp. Maidenii que la Eucalyptus globulus ssp globulus con $13 \pm 23.1$ y $4 \pm 7.7 \%$, respectivamente [21]. La susceptibilidad de los Culex quinquefasciatus colectada en Bucaramanga el AE de Eucalyptus globulus (quimiotipo mentol 27.0\%) fue de $57.7 \mathrm{\mu g} / \mathrm{mL}$ pudiendo considérese este aceite como activo.

Los aceites esenciales estudiados en este trabajo presentaron una mayor bioactividad que los extractos frente a las larvas de Culex quinquefas- 
ciatus, y muestran un alto potencial como nuevos larvicidas, entre los cuales se pueden destacar la $R$. graveolens, O. americanum y C. ambrosioides.

\subsection{Actividad inhibitoria de aceites esencia- les y extractos frente a la enzima acetilcoli- nesterasa}

La inhibición de la acetilcolinesterasa (AChE) es el mecanismo más común de acción insecticida. Para este procedimiento la actividad de la enzima se midió fotométricamente a $412 \mathrm{~nm}$, por el incremento del color amarillo producido por la tiocolina, procedente de la hidrólisis de la acetilcolina, cuando esta reacciona con el ion ditiobisnitrobenzoato para formar el anión del ácido tiobisnitrobenzóico, que es de color amarillo. De esta manera fue posible establecer la actividad acetilcolinesterásica de los extractos y aceites.

Las cuatro muestras más activas fueron: el extracto de $C$. chinense con una $\mathrm{IC}_{50}$ de $18.80 \mu \mathrm{g} /$ $\mathrm{mL}$, seguido por el extracto de $D$. seguine con $19.31 \mu \mathrm{g} / \mathrm{mL}$, por el AE de E. globulus $31.25 \mu \mathrm{g} /$ $\mathrm{mL}$ y el de 0 . americanum con $35.04 \mu \mathrm{g} / \mathrm{mL}$. Para los demás aceites esenciales las concentraciones inhibitorias medias de la enzima estuvieron entre $44-64 \mu \mathrm{g} / \mathrm{mL}$ y para el extracto de $U$. baccifera la $\mathrm{IC}_{50} 37.02 \mu \mathrm{g} / \mathrm{mL}$ (ver tabla III).

Los inhibidores de la AChE, como los insecticidas organofosforados, usados comúnmente para el control de los artrópodos, son altamente tóxicos; pero las larvas, cuando fueron expuestas a los extractos y $\mathrm{AE}$ de origen natural no mostraron los efectos característicos de envenenamiento por inhibición de este target biológico. Muchos de los aceites y extractos no han sido reportados en la literatura como inhibidores de la acetilcolinesterasa, en ningún caso se encontraron comportamientos como inhibidores irreversibles de la enzima, esto es un punto favorable al evitarse el bloqueo de la enzima, lo cual llevaría a una intensa toxicidad aguda y crónica.

\subsection{Toxicidad y cambios fenotípicos en el pez cebra}

Para estudiar la toxicidad de los extractos etanólicos de Capsicum chinense, Dieffenbachia seguine, Urera baccifera y el AE de Ruta graveolens fue empleado el protocolo de Puerto \& Kouznet- sov [16]; se encontró que estos extractos y el $\mathrm{AE}$ son moderadamente tóxicos $\left(10<\mathrm{CL}_{50}<100\right.$ $\mathrm{mg} / \mathrm{L})$ [22]. El extracto de Capsicum chinense presentó una $\mathrm{CL}_{50}$ de $39.7 \mathrm{mg} / \mathrm{L}$, siendo el extracto con menor toxicidad sobre este modelo, seguido por Urera baccifera $33.84 \mathrm{mg} / \mathrm{L}$, Dieffenbachia seguine $32.15 \mathrm{mg} / \mathrm{L}$ y Ruta graveolens $13.33 \mathrm{mg} / \mathrm{L}$. La toxicidad de los extractos y el AE de Ruta graveolens es comparable con moléculas de origen natural, como por ejemplo la teobromina presente en el cacao con concentración letal media de $150.46 \mathrm{mg} / \mathrm{L}$ [222]. Una vez se determinó la $\mathrm{CL}_{50}$, se estableció un rango de concentraciones por debajo de este, para estudiar los cambios fenotípicos en el pez Danio rerio.

En general, todos los embriones tratados con los extractos, a dos o tres concentraciones por debajo de la respectiva $\mathrm{CL}_{50}$, no manifestaron ningún cambio fenotípico visible durante las primeras horas de exposición. Sin embargo, a una o dos concentraciones cercanas a la $\mathrm{CL}_{50}$ se observó un desarrollo anormal de las somitas, que son las responsables de dar lugar a la mayor parte del esqueleto axial y a la musculatura alrededor de la espina dorsal, permitiendo identificar el mecanismo de acción y los efectos tóxicos previos a la muerte del embrión.

Los embriones que presentaron retraso en el desarrollo, finalmente murieron. Sin embargo, aquellos embriones que no presentaron ningún cambio fenotípico visual alcanzaron las etapas posteriores de desarrollo después de $96 \mathrm{hpf}$, sin ningún tipo de evidencia que indicara que su morfología o embriogénesis difiriera de la del control.

Es importante resaltar que la actividad inhibitoria de la AChE por el extracto de $C$. chinense $\left(\mathrm{IC}_{50} 18.80 \mu \mathrm{g} / \mathrm{mL}\right)$ se encuentra por debajo de la $\mathrm{CL}_{50}(39.7 \mu \mathrm{g} / \mathrm{mL})$ en el Danio rerio; al igual que en $D$. seguine $\left(\mathrm{IC}_{50} 19.31 \mu \mathrm{g} / \mathrm{mL} ; \mathrm{CL}_{50} 32.15\right.$ $\mu \mathrm{g} / \mathrm{mL}$ ), de lo que se puede inferir que inhibir la actividad de la enzima no resulta ser letal para el pez, o bien que el mecanismo de acción por el cual el pez muere al ser expuesto al extracto, no está relacionado con la inhibición de colinesterasas, de la misma forma que tampoco causa cambios fenotípicos, daños teratogénicos o malformaciones en el desarrollo. 


\section{CONCLUSIONES}

Se puede considerar que los aceites esenciales y extractos evaluados en esta investigación presentan un alto potencial como larvicidas en Culex quinquefasciatus. De los estudios de inhibición de la enzima acetilcolinesterasa y los ensayos de toxicidad sobre el pez Danio rerio, es posible considerar que estos productos tienen baja toxicidad sobre las colinesterasas de los mamíferos y cambios fenotípicos en el modelo vertebrado del pez cebra.

\section{AGRADECIMIENTOS}

Los autores agradecen a la Universidad Santo Tomás proyecto GIFQCAAMBP42013, y a Colciencias (Proyecto N RC-007-2017, Código: 110274558597) por el apoyo financiero. DNRS agradece a Colciencias por la beca de Joven Investigadora.

\section{REFERENCIAS}

[1] F. Ruiz López, et al., “Presencia de Aedes (Stegomyia) aegypti (Linnaeus, 1762) y su infección natural con el virus del dengue en alturas no registradas para Colombia," Biomédica, vol. 36, no. 2, pp. 303-308, 2016.

[2] V.S.S. Dharmagadda, S.N. Naik, P.K. Mittal, y P. Vasudevan, "Larvicidal activity of Tagetes patula essential oil against three mosquito species," Bioresour. Technol., vol. 96, no. 11, pp. 1235-1240, 2005.

[3] World Health Organization (WHO), "Temephos in drinking-water: Use for vector control in drinking-water sources and containers," pp. 1-15, 2009.

[4] O. Mbare, S. W. Lindsay, y U. Fillinger, "Pyriproxyfen for mosquito control: Female sterilization or horizontal transfer to oviposition substrates by Anopheles gambiae sensu stricto and Culex quinquefasciatus," Parasit Vectors, vol. 7, no. 1, pp. 1-12, 2014.

[5] C.B. Ocampo, M.J. Salazar-Terreros, N.J. Mina, J. McAllister, y W. Brogdon, "Insecticide resistance status of Aedes aegypti in 10 localities in Colombia", Acta Trop., vol. 118, no. 1, pp. 37-44, 2011.

[6] I.S. Rana and A.S. Rana, "Efficacy of essential oils of aromatic plants as larvicide for the management of filarial vector Culex quinquefasciatus Say (Diptera: Culicidae) with special reference to Foeniculum vulgare," Asian Pacific J. Trop. Dis., vol. 2, no. 3, pp. 184-189, 2012.

[7] R.P. Adams, Identification of essential oil components by gas chromatography/quadrupole mass spectroscopy. San Diego, Estados Unidos: Academic press, 1995. 302p.

[8] D. Joliuan y W.A.Köning, The Atlas of Spectral Data of Sesquiterpene Hydrocarbons. Hamburgo, Alemania: EB-Verlag Dr. Brandt e.K.,1998. 661p.
[9] NIST, “NIST Chemistry WebBook," 2017 [en línea] disponible en https://webbook.nist.gov/chemistry/ [Último acceso 18 octubre 2017].

[10] WHO, "Guidelines for laboratory and field testing of mosquito larvicides," World Health. Organazation, pp. 1-41, 2005.

[11] G.L. Ellman, K.D. Courtney, V. Andres, y R.M. Featherstone, "A new and rapid colorimetric determination of acetylcholinesterase activity," Biochem. Pharmacol., vol. 7, no. 2, pp. 88-95, 1961.

[12] S. Ali, H.G. J. van Mil, y M.K. Richardson, "Large-Scale assessment of the zebrafish embryo as a possible predictive model in toxicity testing," PLoS One, vol. 6, no. 6, pp. e21076, 2011.

[13] U.S. Eggert, "The why and how of phenotypic smaII-molecule screens," Nat. Chem. Biol., vol. 9, no. 4, pp. 206-209, 2013.

[14] R.T. Peterson, B.A. Link, J.E. Dowling, y S.L. Schreiber, "Small molecule developmental screens reveal the logic and timing of vertebrate development," Proc. Natl. Acad. Sci., vol. 97, no. 24, pp. 12965-12969, 2000.

[15] A. Rubinstein, "Zebrafish: from disease modeling to drug discovery," Curr. Opin. Drug Discov. Devel., vol. 6, no. 2, pp. 218-223, 2003.

[16] C.E. Puerto Galvis y V.V. Kouznetsov, "An unexpected formation of the novel 7-oxa-2-azabicyclo[2.2.1]hept5 -ene skeleton during the reaction of furfurylamine with maleimides and their bioprospection using a zebrafish embryo model," Org. Biomol. Chem., vol. 11, no. 3, pp. 407-411, 2013.

[17] E.S. Cavalcanti, S.M. Morais, M. A.A. Lima, y E.W.P. Santana, "Larvicidal activity of essential oils from Brazilian plants against Aedes aegypti L.," Mem Inst Oswaldo Cruz, vol. 99, no. 5, pp. 541-544, 2004.

[18] M. Chellappandian, et al., "Botanical essential oils and uses as mosquitocides and repellents against dengue," Environ. Int., vol. 113, no. 113, pp. 214-230, 2018.

[19] B. Conti, M. Leonardi, L. Pistelli, R. Profeti, I. Ouerghemmi, y G. Benelli, "Larvicidal and repellent activity of essential oils from wild and cultivated Ruta chalepensis L. (Rutaceae) against Aedes albopictus Skuse (Diptera: Culicidae), an arbovirus vector", Parasitol. Res., vol. 112, no. 3, pp. 991-999, 2013.

[20] R. Pavela, K. Kaffková, y M. Kumšta, “Chemical composition and larvicidal activity of essential oils from different Mentha L. and Pulegium species against Culex quinquefasciatus Say (Diptera: Culicidae)," Plant Prot. Sci., vol. 50, no. 1, pp. 36-42, 2014.

[21] A. Lucia, S. Licastro, E. Zerba, y H. Masuh, "Yield, chemical composition, and bioactivity of essential oils from 12 species of Eucalyptus on Aedes aegypti larvae," Entomol. Exp. Appl., vol. 129, no. 1, pp. 107-114, 2008.

[22] Environment Directorate (OECD), "Validation Report (Phase 1) for the Zebrafish Embryo Toxicity Test. Part 1," OECD Ser. Test. Assess., vol. 1, no. 157, pp. 1-123, 2011. 